\title{
La consommation collaborative en Europe : démêler la dualité des rôles
}

\author{
Joan Torrent-Sellens ${ }^{a}$, Natàlia Cugueró-Escofet ${ }^{b}$
}

RÉSUMÉ. Cet article apporte de nouvelles preuves sur l'économie collaborative en Europe grâce à l'analyse des motivations à participer à une plateforme collaborative en tant qu'acquéreur ou fournisseur. À cet effet, nous analysons un échantillon paneuropéen de 14050 citoyens provenant de 28 pays. L'étude, qui applique une méthodologie de prévision empirique grâce à un modèle d'équations structurelles, fournit deux principales contributions à la littérature. Premièrement, les motivations économiques et les motivations basées sur l'efficacité prédisent l'acquisition et la fourniture de biens et services sur les plateformes collaboratives en Europe. Deuxièmement, les échanges non financiers prédisent également la fourniture sur les plateformes collaboratives. Nos résultats ont également des incidences sur l'aménagement du territoire. Comprendre les motivations entre les acquéreurs et les fournisseurs peut favoriser les échanges collaboratifs des ressources essentielles, plus particulièrement à petite échelle et à l'échelle locale.

ABSTRACT. Through the analysis of the motivations for participation in collaborative platforms as an obtainer or as a provider, the paper provides new evidence on the collaborative economy in Europe. For that purpose, we analyze a pan-European sample of 14,050 citizens from 28 countries. The study, which applies an empirical prediction methodology through a structural equation modelling (SEM), provides two main contributions to the literature. Firstly, economic and usefulness motivations predict the obtaining and provision of goods and services through collaborative platforms in Europe. Secondly, non-monetary exchanges also predict the provision of collaborative platforms. Our results also have implications for territorial development. Understanding the motivations between obtainers and providers can foster collaborative exchanges of essential resources, especially at a small-scale and at local levels.

\section{Introduction}

L'économie collaborative (EC) ou l'économie de partage peut se définir comme étant «l'ensemble des systèmes de circulation des ressources qui permettent aux consommateurs d'acquérir et de fournir des ressources ou services utiles, de manière temporaire ou permanente, grâce à une interaction directe avec d'autres consommateurs ou par l'intermédiaire d'un médiateur » (Ertz et al., 2016, p. 6). Ainsi, la consommation collaborative (CC) ne concerne pas de simples clients, mais plus spécifiquement des acquéreurs qui peuvent également être des fournisseurs.

Un acquéreur « est un consommateur qui souhaite obtenir une ressource ou un service fourni directement par un autre consommateur (p. ex., le fournis- seur) ou indirectement par l'entremise d'une organisation connue sous l'appellation de "médiateur" (à but lucratif ou sans but lucratif) » (Ertz et al., 2016, p. 5). L'acquisition comprend donc des achats de seconde main, des échanges, un accès gratuit à des ressources ou en échange d'une compensation, ainsi que la consommation de biens restaurés ou remis à neuf (Ertz et al., 2016).

De son côté, un fournisseur « est un consommateur qui fournit un service ou une ressource particulière, soit directement à un consommateur (p. ex., l'acquéreur), soit indirectement par l'intermédiaire d'un médiateur » (Ertz et al., 2016, p. 5). La fourniture fait donc référence à la revente, à l'échange, à l'offre gratuite, à l'accès gratuit à des ressources ou en

\footnotetext{
a Professeur, Université Ouverte de Catalogne (UOC)

${ }^{b}$ Professeure agrégée, Université Ouverte de Catalogne (UOC)
} 
échange d'une compensation, ou au commerce avec une organisation (Ertz et al., 2016).

Cette transition de rôle acquéreur/fournisseur est l'élément essentiel de l'EC (Sutherland et Jarrahi, 2018; Ertz et al., 2019). Cela explique aussi pourquoi l'EC a été considérée comme un facteur contributeur important au travail atypique (p. ex., contractuel, autonome, à temps partiel, temporaire) (Codagnone et al., 2016; Bouncken et Reuschl, 2018), qui est à la hausse partout en Europe (Bardazzi et Duranti, 2012; Kelly et Barrett, 2017). Cette tendance est d'autant plus forte à la suite de la numérisation accélérée causée par la pandémie de la COVID-19, qui permet à plus d'individus d'agir à titre de fournisseurs de ressources en ligne. Pour Nash et al. (2008), cela est même devenu un style de vie tendance qui remet en question les contextes du travail traditionnel.

De plus, même si la littérature foisonne d'études qui analysent les facteurs motivant les intentions ou le recours actuel à l'EC, ces études ne font pas nécessairement la distinction entre les motivations des acquéreurs et celles des fournisseurs (Ertz et al., 2016; Torrent-Sellens, 2019). Pourtant, cela fournirait des informations cruciales qui pourraient modifier les perspectives des chercheurs sur le cadre de motivation sous-jacent aux deux facettes de l'EC. De telles connaissances pourraient également offrir des indications utiles aux gestionnaires afin de concevoir des stratégies optimales pour recruter et fidéliser autant les acquéreurs que les fournisseurs.

Cette étude vise à analyser les facteurs motivationnels et les barrières à l'EC en Europe. Même si les preuves sur les motivations des consommateurs collaboratifs sont assez vastes, nous n'avons actuellement pas assez de résultats sur l'ensemble des acquéreurs et fournisseurs en Europe. Ainsi, pour la première fois, les indicateurs sur l'EC sont analysés conjointement, mais en distinguant leurs impacts respectifs, autant sur les acquéreurs que les fournisseurs, et ce, de manière représentative pour l'ensemble de l'Europe. Les objectifs de recherche et la méthodologie employés peuvent s'appliquer à d'autres territoires ou contextes sociaux, à des formes plus spécifiques d'EC ou pour faire la comparaison entre les plateformes commerciales et non commerciales. À cet égard, les répercussions de cette étude, autant théoriques que managériales, sont vastes, de même que les pistes pour de futures recherches.
Notre recherche présente également un intérêt du point de vue de l'aménagement du territoire. De multiples recherches ont déjà souligné le lien entre la consommation collaborative et le développement durable (Ertz et Leblanc-Proulx, 2018). Par contre, la relation entre la consommation collaborative durable et l'aménagement du territoire a fait l'objet de peu d'études. Nous savons que l'utilisation de grandes plateformes de location de logement génère des problèmes d'embourgeoisement au centre des grandes villes (Wachsmuth et Weisler, 2018). Nous avons également des preuves sur le fait que, localement, ces plateformes sont très utiles pour répondre aux besoins et pour contribuer à l'échange durable des ressources, et que d'autres formes de consommation ne pouvaient pas être mises en place (Cohen et Muñoz, 2016). Des conséquences intéressantes peuvent découler de nos résultats. En ce sens, selon l'identification des motivations différenciées pour les acquéreurs et fournisseurs collaboratifs en Europe, nous en déduisons qu'il existe des impacts pour l'aménagement du territoire.

\section{Analyse de la littérature et hypothèses de recherche}

\subsection{Dualité des rôles et motivations des consommateurs collaboratifs}

Les antécédents de l'EC pourraient différer lorsqu'on agit sur les plateformes collaboratives en tant qu'acquéreur ou en tant que fournisseur. Par exemple, Ertz et al. (2017) trouvent des divergences entre les motivations de premier ordre pour l'acquisition (p. ex., obtention) et la disposition (p. ex., fourniture) de biens matériels. D'ailleurs, les consommateurs motivés principalement par l'une ou l'autre des quatre catégories motivationnelles lors de l'acquisition de biens sont motivés de manière similaire lorsqu'ils se départissent de leurs biens. Toutefois, il est intéressant de noter que les motivations économiques reliées à l'acquisition de biens matériels démontrent une faible association avec les motivations non utilitaires reliées à la fourniture de biens matériels. En d'autres termes, les motivations utilitaires prévalent lors de l'acquisition, mais un ensemble beaucoup plus large de motifs prédit la fourniture.

De la même façon, mais en se servant d'une approche phénoménologique et qualitative, Barbosa et Fonseca (2019) concluent que les avantages perçus de l'EC dépendent du rôle du consommateur collaboratif. 
Même si certaines motivations sociales telles que le sentiment d'appartenance et l'intégration sociale sont pertinentes autant pour les acquéreurs que pour les fournisseurs, d'autres avantages attendus tels que la solidarité, de bonnes actions ou encore les avantages environnementaux ne sont mentionnés que par les fournisseurs.

La littérature perçoit un appui solide à cette prévalence de l'utilitaire, plus particulièrement des motivations économiques, à l'étape de l'acquisition, tandis qu'un plus grand ensemble de motifs non utilitaires importe pour la fourniture (Belloti et al., 2015; Bucher et al., 2016; Wilhelms et al., 2017). Ces résultats démontrent clairement que les deux facettes de l'EC justifient une analyse spécifique.

\subsection{Hypothèses de développement}

Comme la littérature l'a souligné à plusieurs reprises, la participation à des plateformes collaboratives est basée sur la technologie (Belk, 2014; Hamari et al., 2016). Les canaux de l'EC peuvent être purement en ligne (p. ex., le partage de fichiers, les codes sources ouverts, le développement de logiciels) ou ils sont purement hors ligne (p. ex., les marchés aux puces et les ventes-débarras). Pourtant, dans tous les cas, la technologie joue un rôle qui varie en importance. Certains modèles commerciaux reposent entièrement sur des applications et plateformes (p. ex., Uber), c'està-dire une plateforme en ligne en tant que composante principale. Pour d'autres, la technologie améliore la coordination d'activités qui sont essentiellement hors ligne (p. ex., Craigslist), c'est-à-dire des plateformes en ligne en tant que composante facilitatrice. Pour d'autres encore, la technologie (p. ex., un site web, un compte Twitter ou une page Facebook) complémente les opérations hors ligne en offrant des informations utiles et des interactions sociales en ligne (p. ex., l'Armée du Salut), c'est-à-dire une plateforme en ligne en tant que composante mineure (Ertz et al., 2019).

Sans égard à l'importance de ces plateformes, une partie de la prédiction sur l'acquisition et la fourniture en biens et en services par le biais d'une plateforme de pair à pair (peer-to-peer ou P2P) dépend de la capacité des gens à apprendre à utiliser ces plateformes et à s'en servir (Hawlitschek et al., 2018). Cela implique de savoir que l'EC existe et de posséder des connaissances sur son utilisation (Gazzola et al., 2018; Vătămănescu et Pînzaru, 2018). Ainsi, nous énonçons l'hypothèse 1 suivante :
H1: La connaissance de l'existence des plateformes prédit de manière positive la participation des acquéreurs sur les plateformes collaboratives.

La recherche sur la dualité des rôles des consommateurs collaboratifs démontre une forte concordance entre les motivations pour l'obtention (acquéreurs) et la disposition (fournisseurs) (Ertz et al., 2017). Ainsi, une fois que le comportement général du participant collaboratif a été identifié, la structure motivationnelle des acquéreurs et des fournisseurs s'avère assez semblable. Cela peut inciter au changement du statut d'acquéreur à fournisseur, et vice versa (Ertz et al., 2017). De plus, et considérant les récentes recherches sur la production participative (crowdsourcing) en Europe (Huws et al., 2017; Pesole et al., 2018) qui mettent en évidence un lien statistique important entre les fournisseurs et les acquéreurs, il est possible d'émettre l'hypothèse que l'entrée dans les plateformes collaboratives s'effectue normalement par l'acquisition de biens et services. Cela conduira par la suite à une participation en tant que fournisseur. Ainsi, nous proposons l'hypothèse 2 :

$\mathbf{H} 2$ : Une participation en tant qu'acquéreur sur une plateforme collaborative prédit de manière positive une participation en tant que fournisseur.

Toutefois, les processus de motivation des acquéreurs et des fournisseurs ne sont pas entièrement symétriques (Ertz et al., 2017). L'aspect le plus intéressant est de constater que les motivations utilitaires des acquéreurs ne sont pas automatiquement transférées aux fournisseurs, qui eux ne participent pas nécessairement à la plateforme collaborative pour des raisons utilitaires (Ertz et al., 2017). La recherche initiale sur l'EC révèle déjà l'importance de ces motivations alternatives (Brodie et al., 2013), soit l'anticonsommation (Ozanne et Ballantine, 2010) et l'antimatérialisme (Albinsson et Perera, 2012). Il convient de souligner que ces études étaient axées sur des systèmes prosociaux, tels que les ludothèques (Ozanne et Ballantine, 2010) et les marchés alternatifs tels que Really Really Free Marketplaces (RRFMs).

Des recherches plus récentes qui comparent les motivations des acquéreurs et des fournisseurs révèlent également des résultats divergents entre ces derniers. Belloti et al. (2015) concluent que les motivations des fournisseurs diffèrent de celles des acquéreurs. Des idéologies telles que construire une meilleure communauté grâce à des échanges non 
commerciaux et grâce à l'augmentation du développement durable justifient la participation du fournisseur, tandis que des raisons d'ordre pratique telles que la satisfaction, la valeur ajoutée et la commodité justifient la participation de l'acquéreur. Bucher et al. (2016) déterminent que l'attitude de l'acquéreur collaboratif est liée à des motivations morales, sociales et financières. De même, les incitations financières sont considérées comme étant nécessaires, mais non comme une condition suffisante pour fournir des biens individuels à d'autres personnes. Wilhelms et al. (2017) estiment que les motivations individuelles financières et basées sur la qualité de vie ne prédisent pas à elles seules la fourniture des plateformes collaboratives de covoiturage. En fait, les fournisseurs souhaitent également aider les gens en offrant de la mobilité, non seulement en échange d'une compensation financière. Le développement durable n'est pas la motivation principale, mais elle en est aussi une conséquence indirecte. Ainsi, nous énonçons l'hypothèse 3 :

H3 : Les échanges non financiers prédisent de manière positive la fourniture des plateformes collaboratives.

La littérature initiale en gestion sur l'EC a également souligné un nombre d'aspects économiques qui pourraient encourager la participation sur les plateformes collaboratives. Ces motivations peuvent être rationnelles, soit adopter un comportement d'utilité ou d'optimisation de l'utilité. C'est le cas, par exemple, lorsqu'un consommateur remplace une possession exclusive et coûteuse par des utilisations à faible coût ou économise des ressources financières grâce à l'EC (Tussyadiah, 2015, 2016). Cette littérature initiale a aussi prouvé l'importance des motivations basées sur l'aspect commode, pratique et utile (Hamari et al., 2016). Möhlmann (2015) constate que les avantages perçus expliquent la satisfaction de l'acquéreur et la probabilité de réutilisation de ces plateformes. L'utilité, la confiance, la réduction des coûts et la familiarité ont été des facteurs déterminants pour les deux échantillons de consommateurs collaboratifs. Balck et Cracau (2015) concluent que les prix inférieurs sont la motivation principale des acquéreurs. La rareté, l'environnement et l'accès à la propriété constituent également des facteurs importants. Barnes et Mattsson (2017) confirment que leurs conceptions des motivations économiques (gain ou économie d'argent, options à bas prix et bon rapport qualitéprix) et des motivations basées sur l'utilité perçue (bénéfices et avantages pour le participant) prédisent les intentions de participer à un site web de covoiturage au Danemark et de le recommander.

Hawlitschek et al. (2018) constatent que les avantages financiers jouent un rôle motivationnel majeur. Économiser de l'argent, réduire les dépenses et vivre raisonnablement font partie de ces motivations économiques. Gazzola et al. (2018) constatent également que les avantages économiques (p. ex., avoir accès à de bas prix, vivre judicieusement, réduire le nombre de biens non essentiels acquis, faire de l'argent grâce à des objets ou encore générer un revenu additionnel en offrant des produits qui ne servent pas) prédisent le degré de participation et le nombre de résultats obtenus par les participants des plateformes collaboratives de pair à pair.

Park et Armstrong (2019) déterminent quant à eux cinq motivations fondamentales: économiser de l'argent, économiser du temps, trouver les produits convoités, avoir une utilité et ne pas porter le fardeau de la propriété. Dans la même lignée, Li et Wen (2019) concluent que les avantages économiques perçus (économiser de l'argent, réduire les coûts, réduire le temps de transport et être plus disponible) et la commodité (accès en tout temps de partout, utilisation simple et contribution à l'environnement) prédisent l'utilité perçue, qui, en retour, agit en tant que médiateur des intentions de l'acquéreur à participer à des plateformes de vélos de pair à pair. Ainsi, une première série d'hypothèses (H4a-c) positionne les motivations reliées au prix, à la nouveauté et à la commodité. La quatrième hypothèse $(\mathrm{H} 4 \mathrm{~d})$ prédit les effets des motivations non financières pour les acquéreurs, qui offrent déjà leurs services comme fournisseurs (H3). De manière similaire, nous proposons également une autre série d'hypothèses (H5a-c) relatives aux fournisseurs (Wilhelms et al., 2017) :

H4a/H5a : Le prix prédit positivement l'acquisition/la fourniture sur les plateformes collaboratives.

H4b/H5b : La nouveauté prédit positivement l'acquisition/la fourniture sur les plateformes collaboratives.

H4c/H5c : La commodité prédit positivement l'acquisition/la fourniture sur les plateformes collaboratives.

H4d : Les échanges non financiers prédisent positivement l'acquisition sur des plateformes collaboratives. 
Concernant les obstacles à la participation aux plateformes collaboratives, nous avons identifié divers résultats dans la littérature. D'abord, Tussyadiah (2015) explore les obstacles à l'EC grâce à un échantillon de participants de plateformes d'hébergement de pair à pair. L'étude démontre que le manque de confiance, d'efficacité et d'avantages économiques constitue les principaux obstacles. Hawlitschek et al. (2016) identifient les risques procéduraux d'une participation à une plateforme de pair à pair, la perte de vie privée et le manque de confiance entre les participants comme étant des obstacles à l'EC.

Toutefois, les plus récentes recherches systématisent et organisent cet ensemble d'obstacles. Barnes et Mattsson (2017) constatent que les conditions de sécurité (protection, garanties et identification des participants) par l'intermédiaire de la plateforme prédisent la confiance (honnêteté, souci des usagers, prévision et connaissance du marché) des acquéreurs, ce qui en retour agit comme un motivateur des intentions de recommander leur usage.

De leur côté, Hawlitschek et al. (2018) définissent et évaluent quatre concepts : 1) préoccupations reliées aux risques du processus (p. ex., risques économiques, légaux et individuels sur les plateformes de pair à pair; Hawlitschek et al., 2016); 2) préoccupations relatives à la vie privée (p. ex., visibilité et divulgation des informations et données personnelles; Lutz et al., 2018; Teubner et Flath, 2019); 3) anticipations des efforts (difficulté et familiarisation avec l'utilisation des plateformes collaboratives; Edbring et al., 2016); et 4) confiance (p. ex., assurance, engagement et considération de l'intérêt personnel des autres usagers des plateformes collaboratives; Hawlitschek et al., 2018; Ter Huurne et al., 2017). Leurs résultats indiquent que la méfiance envers autrui, l'anticipation des efforts et, dans une moindre mesure, les préoccupations reliées aux risques du processus limitent l'intention d'agir. Par conséquent, selon la théorie du comportement planifié (TCP), elles limitent également le comportement d'acquisition sur les plateformes collaboratives.
Finalement, dans une recherche qualitative axée uniquement sur les obstacles à l'EC, Spindeldreher et al. (2019) identifient une série de facteurs nuisibles autant aux acquéreurs qu'aux fournisseurs. Alors que certains d'entre eux sont reliés à l'utilisation des plateformes par les acquéreurs et les fournisseurs (p. ex., anticipations des efforts, exploitation, manque de confiance, risques reliés à la vie privée ou aux processus), d'autres se rapportent à l'échange de ressources (p. ex., inflexibilité, risques reliés à la performance, risques physiques, risques dus au processus d'acquisition, anticipations reliées aux efforts et risques reliés au processus pour les fournisseurs). D'autres encore portent sur la relation établie avec les autres participants des plateformes de pair à pair (p. ex., manque de confiance, risques physiques, risques reliés à la vie privée et interaction sociale non désirée).

Ainsi, nos sixième et septième hypothèses de travail concernent les obstacles de préoccupation reliés aux risques du processus et à la méfiance - comme perçus par les acquéreurs et les fournisseurs - puisqu'ils semblent tous les deux êtres des obstacles récurrents dans diverses études. La première série d'hypothèses (H6a-c et H7a-c) fait référence aux obstacles liés aux préoccupations sur les risques pour le participant, tandis que les deux dernières (H6d-e et H7d-e) traitent de la méfiance des participants :

H6a/H7a : L'absence d'une personne responsable prédit de manière négative l'acquisition/la fourniture sur les plateformes collaboratives.

H6b/H7b : L'absence de réponse aux attentes en matière de service prédit de manière négative l'acquisition/la fourniture sur les plateformes collaboratives.

H6c/H7c: Le manque d'information prédit de manière négative l'acquisition/la fourniture sur les plateformes collaboratives.

H6d/H7d : Le manque de confiance envers les autres prédit de manière négative l'acquisition/la fourniture sur les plateformes collaboratives.

H6e/H7e : Le manque de confiance envers Internet prédit de manière négative l'acquisition/la fourniture sur les plateformes collaboratives. 


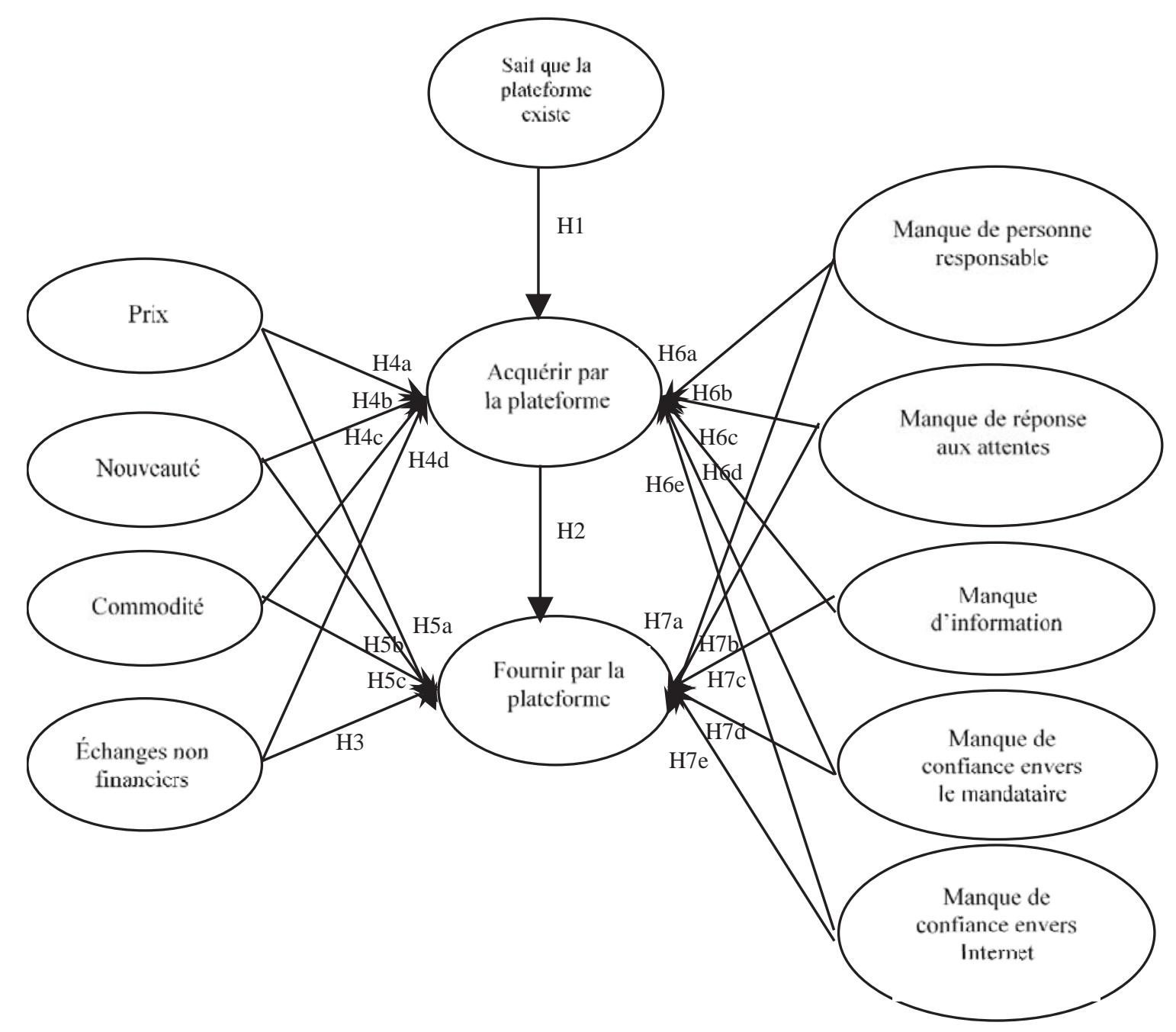

Figure 1 - Modèle empirique des motivations et obstacles à participer aux plateformes collaboratives de pair à pair

\section{Méthodologie}

\subsection{Collecte et mesure des données}

Nous employons le sondage de la Commission Européenne (2016a, 2016b) Flash Eurobarometer (numéro 438) portant sur l'utilisation des plateformes d'EC. Au moyen d'entrevues téléphoniques, le Flash Eurobarometer 438 a obtenu des informations sur l'utilisation des plateformes d'EC auprès d'un échantillon de 14050 citoyens âgés de 15 ans et plus, dans 28 pays de l'Union européenne, par le biais d'environ 500 entrevues par pays. La composition de l'échantillonnage pour chaque pays était probabiliste et représentative. La marge d'erreur pour la confiance à $95 \%$ était de
0,4\% pour l'ensemble de l'échantillon et d'environ $1,9 \%$ pour les échantillons individuels de chaque pays. Le travail sur le terrain a été effectué les 15 et 16 mars 2016.

Le questionnaire définit la plateforme collaborative (PC) comme étant « un outil Internet permettant d'effectuer des transactions entre des gens qui fournissent et utilisent un service. Elle sert pour une large gamme de services, de la location d'hébergement ou de voiture à des services de travaux ménagers » (Commission Européenne, 2016b, p. 29). Selon cette définition, le sondage demandait aux répondants s'ils connaissaient de telles plateformes et leur donnait les 
options suivantes comme réponses possibles pour l'acquisition :

1) Ne connait pas ou «n'a jamais entendu parler de ces plateformes $»$;

2) Connait, mais n'acquiert pas ou «a entendu parler de ces plateformes, mais ne les a jamais visitées »;

3) Première acquisition ou «a visité une ou plusieurs de ces plateformes et a payé une fois pour un service »;

4) Acquisition occasionnelle ou « utilise les services de ces plateformes occasionnellement (quelques fois par an) ॥;

5) Utilisateur régulier ou "utilise régulièrement les services de ces plateformes (au moins une fois par mois)».

Pour tous les acquéreurs de telles plateformes, ce qui inclut une première acquisition, une utilisation occasionnelle ou régulière, le sondage a également recueilli des données sur la fourniture de biens et services. Il a donné aux répondants les options suivantes comme réponses possibles :

1) Ne fournit pas ou «non, n'a pas fourni »;

2) A fourni une fois ou «a offert un service sur l'une ou plusieurs de ces plateformes une fois »;

3) Fournisseur occasionnel ou « offre des services sur ces plateformes de manière occasionnelle (quelques fois par an) »;

4) Fourniture régulière ou « offre des services régulièrement sur ces plateformes (chaque mois) ».

Les différentes options de ces deux variables ont été transformées en variables individuelles. Toutes ces nouvelles variables individuelles étaient dichotomiques, où $1=$ le répondant connaissait la ou les plateformes et a obtenu ou fourni des biens ou des services sur les plateformes collaboratives, et $0=$ le répondant a répondu autrement.

Après avoir précisé les niveaux d'acquisition et de fourniture, le sondage examinait les motivations et obstacles à l'utilisation des plateformes collaboratives par les acquéreurs et les fournisseurs. Concernant les facteurs motivationnels, le sondage a donné aux répondants et participants (acquéreurs et fournisseurs) qui connaissaient les plateformes collaboratives les options suivantes comme réponses possibles :
1) Prix du service ou "c'est moins cher ou c'est gratuit »;

2) Nouveauté du service ou « un service différent ou nouveau est offert»;

3) Commodité du service ou «l'accès au service est organisé de façon plus pratique »;

4) Échanges non financiers ou «la capacité d'échanger des produits ou des services au lieu de payer avec de l'argent».

Concernant les obstacles, le sondage a donné aux répondants et participants (acquéreurs et fournisseurs) qui connaissaient les plateformes collaboratives les options suivantes comme réponses possibles:

1) Manque de personne responsable lorsque survient un problème ou " ne sait pas qui est responsable en cas de problème »;

2) Manque de satisfaction des attentes en matière de services ou «a été déçu, car les services ou les biens ne satisfaisaient pas les attentes ";

3) Manque d'information ou «n'a pas assez d'information sur les services fournis ";

4) Manque de confiance envers les mandataires ou « ne fait pas confiance au fournisseur ou vendeur »;

5) Manque de confiance envers Internet ou « ne fait pas confiance aux transactions effectuées sur Internet en général ».

Toutes ces variables étaient dichotomiques, où $1=\mathrm{le}$ répondant a répondu positivement aux motivations ou aux obstacles, et $0=$ le répondant a répondu autrement.

\subsection{Statistiques descriptives}

Le sondage a révélé que plus de la moitié des citoyens européens ne connaissaient pas ces nouvelles formes de consommation $(53,2 \%)$, alors qu'un tiers les connaissait, mais n'y avait jamais participé (33,9\%). Ainsi, seulement 12,9\% de l'échantillon a déclaré être acquéreur. Parmi ces derniers $(12,9 \%)$, près des trois quarts n'avaient jamais fourni aucune ressource $(72,1 \%)$. Par conséquent, seulement 3,6\% de l'échantillon a fourni une ressource. Les statistiques détaillées sont présentées au tableau 1. 


\begin{tabular}{|c|c|c|c|}
\hline Motivations et obstacles des participants & $\%$ & \multicolumn{2}{|l|}{ Caractéristiques sociodémographiques } \\
\hline Connaît et utilise & $(13837)$ & $\hat{A g e}$ & $(14050)$ \\
\hline Ne connaît pas & 53,2 & Âge (continu $)^{2}$ & 54,3 \\
\hline Connaît, mais n'utilise pas & 33,9 & 15 à 24 ans & 6.0 \\
\hline Première acquisition & 3,2 & 25 à 34 ans & 9,3 \\
\hline Acquisition occasionnelle & 6,5 & 35 à 44 ans & 14,2 \\
\hline Acquisition régulière & 3,2 & 45 à 54 ans & 18,0 \\
\hline \multirow[t]{2}{*}{ Acquisition totale ${ }^{1}$} & 12,9 & 55 à 64 ans & 20,4 \\
\hline & & 65 ans et plus & 32,2 \\
\hline Fourniture de biens et services & $(1778)$ & & \\
\hline N’a jamais fourni & 72,1 & Genre & $(14050)$ \\
\hline A fourni une fois & 7,3 & Homme & 41,6 \\
\hline Fourniture occasionnelle & 15,7 & Femme & 58,4 \\
\hline Fourniture régulière & 5,0 & & \\
\hline \multirow[t]{2}{*}{ Fourniture totale ${ }^{1}$} & 27,9 & Capital humain (années d'études) & $(13655)$ \\
\hline & & Toujours aux études & 4,3 \\
\hline Facteurs motivationnels & $(6477)$ & Jusqu'à 15 ans & 13,2 \\
\hline Prix & 31,4 & 16 à 19 ans & 39,0 \\
\hline Nouveauté & 22,4 & 20 ans ou plus & 43,4 \\
\hline Commodité & 39,1 & & \\
\hline \multirow[t]{2}{*}{ Échange non financier } & 21,8 & Situation professionnelle & $(13821)$ \\
\hline & & Autonome et gens d'affaires & 8,7 \\
\hline Obstacles & $(6477)$ & Employé - Directeur & 6,3 \\
\hline Manque de personne responsable & 36,5 & Employé - Professionnel qualifié & 5,6 \\
\hline Manque de réponse aux attentes & 25,9 & Employé - Travailleur non manuel & 20,3 \\
\hline Manque d'information & 18,6 & Employé - Travailleur manuel & 6,4 \\
\hline Manque de confiance envers le mandataire & e 25,0 & Sans emploi - Parent/gens de carrière & 7,2 \\
\hline \multirow[t]{17}{*}{ Manque de confiance envers Internet } & 27,2 & Sans emploi - Étudiant & 4,0 \\
\hline & & Sans emploi - Retraité & 37,3 \\
\hline & & Sans emploi (recherche d'emploi) & 4,1 \\
\hline & & Membres du ménage & (13 938) \\
\hline & & Un & 24,3 \\
\hline & & Deux & 44,0 \\
\hline & & Trois & 16,7 \\
\hline & & Quatre ou plus & 15,1 \\
\hline & & Région & (13 908) \\
\hline & & Village ou zone rurale & 32,0 \\
\hline & & Petite ou moyenne ville & 37,2 \\
\hline & & Grande ville ou zone métropolitaine & 30,7 \\
\hline & & Groupe de pays & $(14050)$ \\
\hline & & Europe continentale ${ }^{3}$ & 21,5 \\
\hline & & Europe méditerranéenne ${ }^{4}$ & 25,0 \\
\hline & & Europe du Nord ${ }^{5}$ & 17,9 \\
\hline & & Europe centrale et de $1^{\prime} E_{s t}{ }^{6}$ & 35,7 \\
\hline
\end{tabular}

Note. Le nombre de participants est entre parenthèses.

1. Somme des acquéreurs et fournisseurs de premier usage, d'usage occasionnel et d'usage régulier.

2. Moyen.

3. Europe continentale : Belgique, France, Luxembourg, Pays-Bas, Autriche et Allemagne.

4. Europe méditerranéenne : Grèce, Espagne, Italie, Portugal, Chypre, Malte et Croatie.

5. Europe du Nord : Danemark, Finlande, Suisse, Royaume-Uni et Irlande.

6. Europe centrale et de l'Est : Bulgarie, République tchèque, Estonie, Hongrie, Lettonie, Lituanie, Pologne,

Roumanie, Slovénie et Slovaquie.

Tableau 1 - Motivations et caractéristiques sociodémographiques des participants de l'échantillon 


\section{Résultats}

\subsection{Modèle global}

Nous utilisons le modèle d'équations structurelles (structural equation modeling ou SEM) selon un ensemble de variables observées provenant de l'ensemble des données d'origine. Nous testons un modèle général qui considère l'échantillon comme un seul groupe. Le tableau 2 et la figure 2 présentent les résultats (coefficients des effets directs). Le modèle s'est avéré adapté $($ RMSEA $=0,08 ;$ TLI $=0,889$ et CFI $=0,981)$.

\begin{tabular}{|c|c|c|c|c|c|c|}
\hline \multirow{2}{*}{$\begin{array}{l}\text { Co } \\
\text { Acquéreurs (N = 1 792) }\end{array}$} & befficient & \multirow[t]{2}{*}{ S. E. } & \multirow[t]{2}{*}{$\mathrm{z}$} & \multirow[t]{2}{*}{$P>|z|$} & \multicolumn{2}{|c|}{ [95\% Conf. int.] } \\
\hline & & & & & & \\
\hline Constante & 0,000 & 0,010 & 0,00 & 1000 & {$[-0,021$} & $0,021]$ \\
\hline Connait les plateformes & 0,178 & 0,013 & 13,50 & 0,000 & {$[0,152$} & $-0,204]$ \\
\hline Prix & 0,165 & 0,009 & 18,86 & 0,000 & {$[0,148$} & $-0,182]$ \\
\hline Nouveauté & 0,082 & 0,009 & 9,54 & 0,000 & {$[0,065$} & $-0,099]$ \\
\hline Commodité & 0,244 & 0,009 & 26,55 & 0,000 & {$[0,225$} & $-0,262]$ \\
\hline Échange non financier & $-0,001$ & 0,008 & $-0,17$ & 0,866 & {$[-0,018$} & $-0,015]$ \\
\hline Manque - pers. responsable & $-0,010$ & 0,009 & $-1,10$ & 0,271 & {$[-0,029$} & $0,008]$ \\
\hline Manque - réponse attentes & 0,022 & 0,009 & 2,53 & 0,012 & {$[0,005$} & $0,040]$ \\
\hline Manque - information & $-0,001$ & 0,008 & $-0,15$ & 0,880 & {$[-0,018$} & $0,155]$ \\
\hline Manque - conf. mandataire & 0,017 & 0,009 & 1,91 & 0,046 & {$[0,000$} & $0,034]$ \\
\hline Manque - confiance Internet & $-0,168$ & 0,010 & $-1,87$ & 0,061 & {$[-0,034$} & $0,001]$ \\
\hline Fournisseurs $(\mathrm{N}=496)$ & & & & & & \\
\hline Constante & $-0,001$ & 0,009 & $-0,15$ & 0,878 & {$[-0,020$} & $0,017]$ \\
\hline Utilisateur & 0,498 & 0,007 & 67,86 & 0,000 & {$[0,484$} & $0,513]$ \\
\hline Prix & 0,017 & 0,008 & 2,02 & 0,043 & {$[0,001$} & $0,034]$ \\
\hline Nouveauté & 0,018 & 0,008 & 2,17 & 0,030 & {$[0,002$} & $0,034]$ \\
\hline Commodité & 0,009 & 0,009 & 1,01 & 0,314 & {$[-0,009$} & $0,027]$ \\
\hline Échange non financier & 0,033 & 0,008 & 4,01 & 0,000 & {$[0,017$} & $0,049]$ \\
\hline Manque - pers. responsable & $-0,043$ & 0,009 & $-4,90$ & 0,000 & {$[-0,060$} & $-0,026]$ \\
\hline Manque - réponse attentes & 0,010 & 0,008 & 1,23 & 0,220 & {$[-0,006$} & $0,026]$ \\
\hline Manque - information & $-0,011$ & 0,008 & $-1,41$ & 0,158 & {$[-0,026$} & $0,004]$ \\
\hline Manque - conf. mandataire & $-0,007$ & 0,008 & $-0,89$ & 0,371 & {$[-0,023$} & $0,009]$ \\
\hline Manque - confiance Internet & $-0,013$ & 0,009 & $-1,63$ & 0,103 & {$[-0,029$} & $0,003]$ \\
\hline
\end{tabular}

Note. Analyse des indicateurs : analyse des relations du modèle d'équations structurelles. Coefficients estimés : effets directs.

Tableau 2 - Prédictions des acquisitions et fournitures sur les plateformes collaboratives de pair à pair en Europe

H1 est validée puisque connaitre l'existence des plateformes prédit de manière positive la participation des acquéreurs. H2 est également validée et la participation en tant qu'acquéreurs sur les plateformes collaboratives prédit une participation en tant que fournisseurs. Les résultats soutiennent $\mathrm{H} 3$ puisque les échanges non financiers prédisent de manière positive le rôle des fournisseurs sur les plateformes. Concernant $\mathrm{H} 4-5$, nous constatons que le prix (H4a), la nouveauté $(\mathrm{H} 4 \mathrm{~b})$ et la commodité $(\mathrm{H} 4 \mathrm{c})$ prédisent de manière positive la participation en tant qu'acquéreurs. Toutefois, les échanges non financiers $(\mathrm{H} 4 \mathrm{~d})$ ne prédisent pas une participation en tant qu'acquéreurs (alors que c'était le cas pour la fourniture en H3). Fait intéressant, le prix (H5a) et la nouveauté (H5b) prédisent seulement de manière marginale la participation en tant que fournisseurs, alors que la commodité (H5c) ne prédit pas une participation en tant que fournisseurs. Collectivement, ces résultats soutiennent H4a-c, alors qu'ils ne soutiennent pas H4d et H5a-c. 
De plus, nous constatons que le manque de réponse aux attentes (H6b) et le manque de confiance envers le mandataire (H6d) prédisent de manière négative la participation en tant qu'acquéreurs.

Enfin, nous constatons que le manque de personne responsable $(\mathrm{H} 7 \mathrm{a})$ fait obstacle à la participation en tant que fournisseurs. Toutefois, ce signe négatif du coefficient obtenu renverse l'hypothèse proposée. Selon ces résultats, le manque de personne responsable de la plateforme deviendrait une motivation pour la fourniture de biens et services sur les plateformes de pair à pair. Pourtant, les résultats n'ont pas soutenu le manque de personne responsable (H6a) et le manque d'information (H6c), qui sont tous deux limitants pour l'acquéreur, ni non plus le manque de réponse aux attentes $(\mathrm{H} 7 \mathrm{~b})$ et le manque d'information $(\mathrm{H} 7 \mathrm{c})$, qui sont limitants pour le fournisseur.

Par ailleurs, le manque de confiance (envers les mandataires ou envers Internet) n'a pas d'incidence entravante sur la participation en tant que fournisseurs sur la plateforme (H7d, H7e). De son côté, le manque de confiance envers Internet ne prédit pas la participation en tant qu'acquéreurs sur la plateforme (H6e).

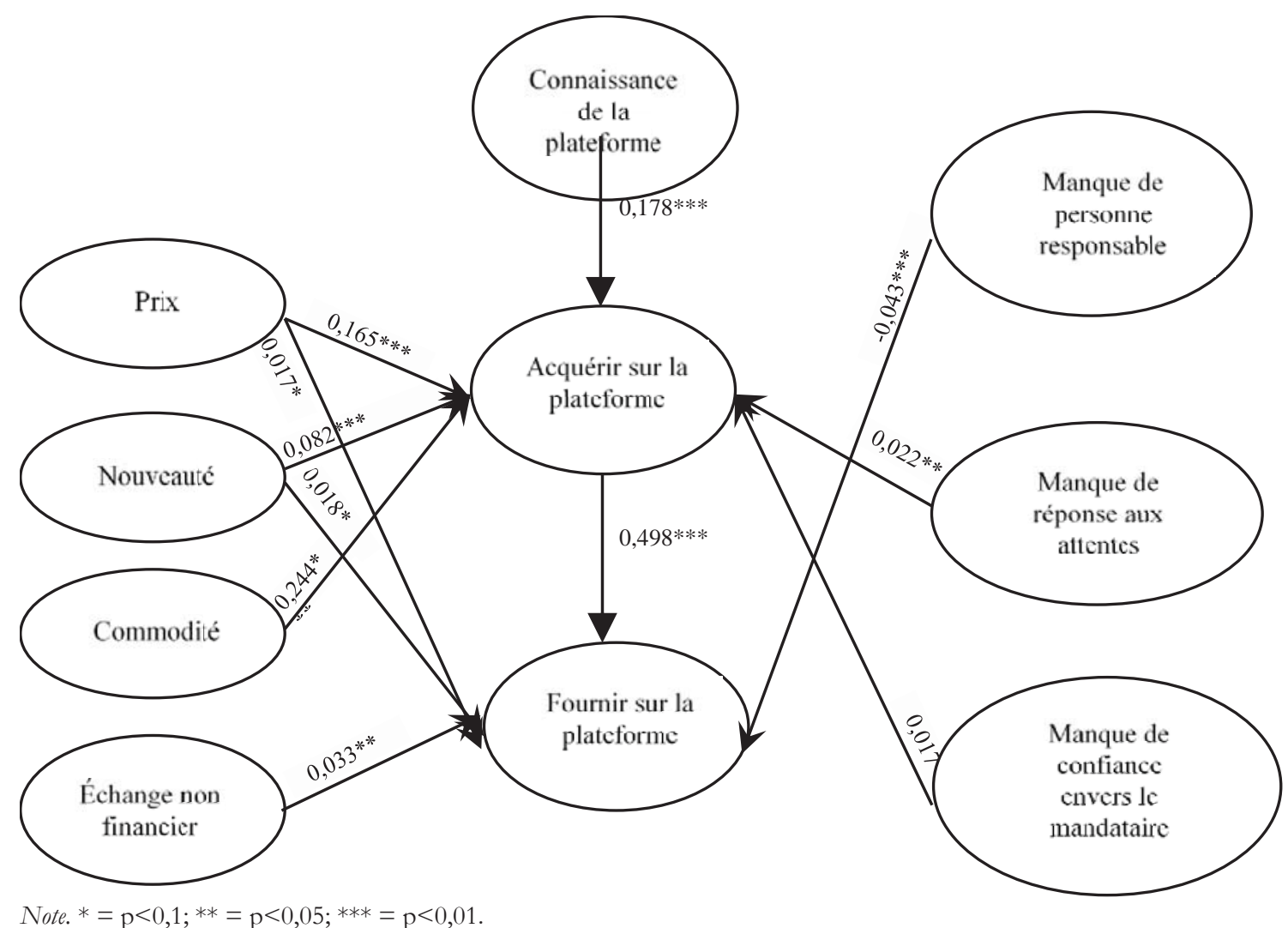

Figure 2 - Indicateurs de la participation aux plateformes collaboratives de pair à pair (effets directs)

\section{Discussion et conclusion}

Au sein de l'économie collaborative, la consommation collaborative est définie comme étant un système de circulation des ressources (temporaire ou permanent, avec une interaction directe ou avec des intermédiaires), essentiellement par le biais des plateformes de pair à pair qui encouragent la dualité des rôles (Ertz et al., 2016, 2017). Plus précisément, et allant au-delà des types d'échanges sur les plateformes et de leur orientation commerciale, cette dualité des rôles, qui permet aux consommateurs collaboratifs de participer en tant qu'acquéreurs ou fournisseurs de biens et services, est si distinctive de la consommation traditionnelle qu'elle justifie une analyse détaillée de ses différents facteurs motivationnels (Ertz et Leblanc-Proulx, 2018). 
Au moyen d'une analyse par modèle d'équations structurelles, notre recherche a défini un ensemble de forces qui prédisent l'acquisition et la fourniture de ressources sur les plateformes collaboratives en Europe. Concernant les acquéreurs, le modèle prévisionnel obtenu décrit un schéma de motivation incluant des facteurs de connaissance des plateformes collaboratives (savoir qu'elles existent), des motivations économiques et liées à l'utilité (prix, nouveauté, commodité) et des obstacles causés par les préoccupations en lien avec les risques du processus (manque de réponse aux attentes) et par le manque de confiance envers les autres mandataires du système d'échange collaboratif.

Ces motivations et obstacles, largement attestés par la littérature (Barnes et Mattsson, 2017; Hawlitschek et al., 2018), soulignent l'importance de stimuler un ensemble de facteurs pouvant motiver la consommation collaborative en Europe. Ces modèles courants, selon lesquels les stratégies d'affaires ou les nouveaux modèles commerciaux devraient agir, seraient reliés à deux aspects fondamentaux :

\section{L'amélioration des connaissances sur le fonctionne-} ment, les outils et la sécurité des plateformes: Une meilleure connaissance du potentiel et de la sécurité des plateformes attirerait plus de personnes vers la consommation collaborative;

2. Une visibilité renforcée des aspects économiques et de l'utilité que les plateformes collaboratives offrent à ses acquéreurs: Les échanges à un plus bas prix, la nouveauté et la commodité de ce type d'échange sont également des éléments fondamentaux pour attirer des consommateurs vers ces nouvelles formes d'échanges.

Concernant les fournisseurs, le modèle prévisionnel obtenu décrit un schéma de motivation qui inclut le précédent rôle d'acquéreur, les motivations économiques et liées à l'utilité (prix et nouveauté), la possibilité de faire des échanges non financiers, l'absence de barrières reliées aux préoccupations en lien avec les risques du processus et le manque de confiance. Sur la base de la littérature antérieure qui insiste sur les motivations explicites envers l'économie collaborative (Barbosa et Fonseca, 2019; Bucher et al., 2016; Ertz et al., 2017; Spindeldreher et al., 2019; Wilhelms et al., 2017), les résultats soulignent la pertinence des actions précédentes en tant qu'acquéreur. L'expérience préalable de l'utilisation des plateformes, la perception de l'utilité de cette expérience et les effets du réseau établi parmi les participants sont essentiels au développement de nouveaux modèles collaboratifs commerciaux (Dreyer et al., 2017; Stofberg et Bridoux, 2019).

Notre recherche souligne également l'existence d'un schéma motivationnel qui allie les facteurs économiques et liés à l'utilité (prix et nouveauté) aux facteurs liés à la possibilité d'effectuer des échanges non financiers. La transition vers la fourniture collaborative doit tenir compte de cette dualité motivationnelle des fournisseurs. Il est très probable que le résultat de l'expérience cumulée comme participant sur la plateforme fasse en sorte que les fournisseurs ne voient plus les risques et le manque de confiance comme un obstacle important à leur participation aux plateformes de pair à pair. Leur expérience acquise précédemment en tant qu'acquéreur réduit l'importance des barrières motivationnelles et suggère qu'on doive encourager la participation en tant qu'acquéreur comme première expérience avant celle de fournisseur.

Enfin, nos résultats indiquent une incidence sur l'aménagement du territoire. Des recherches précédentes ont souligné les effets positifs et négatifs de la consommation collaborative sur le territoire (Cohen et Muñoz, 2016; Wachsmuth et Weisler, 2018). Globalement, les facteurs externes négatifs seraient liés à la substitution des activités traditionnelles ou aux problèmes de logement et de saturation des zones plus touristiques. Les facteurs externes positifs seraient liés à des augmentations additionnelles du revenu, à plus de formes d'échanges durables ou à la possibilité d'accéder à des échanges et des modes de vie auparavant inenvisageables. Afin de minimiser les facteurs négatifs et d'augmenter les facteurs positifs, il est très important de connaître les différentes motivations des acquéreurs et fournisseurs. Par exemple, en Europe, les problèmes de congestion et de saturation pourraient être atténués en satisfaisant les motivations pratiques ou utiles grâce à d'autres types d'hébergement ou d'autres zones touristiques moins achalandées. D’un autre côté, les facteurs externes positifs pourraient être promus grâce à la promotion d'échanges collaboratifs prosociaux locaux. 


\section{RÉFÉRENCES}

Albinsson, P. A. et Perera, B. Y. (2012). Alternative marketplaces in the 21st century: Building community through sharing events. Journal of Consumer Behaviour, 11(4), 303-315. https://doi.org/10.1002/cb.1389

Balck, B. et Cracau, D. (2015). Empirical analysis of customer motives in the share economy: A cross-sectoral comparison. Magdebourg, Allemagne: Otto von Guericke University Magdeburg, Faculty of Economics and Management. Repéré à https://ideas.repec.org/p/mag/wpaper/150002.html

Barbosa, B. et Fonseca, I. (2019). A phenomenological approach to the collaborative consumer. Journal of Consumer Marketing, 36(6), 705-714. https://doi.org/10.1108/JCM-11-2017-2468

Bardazzi, R. et Duranti, S. (2012). Atypical contracts and Italian firms' labour productivity. Repéré à http://hdl.handle.net/2158/777231

Barnes, S. J. et Mattsson, J. (2017). Understanding collaborative consumption: Test of a theoretical model. Technological Forecasting and Social Change, 118, 281-292. https://doi.org/10.1016/j.techfore.2017.02.029

Belk, R. W. (2014). You are what you can access: Sharing and collaborative consumption online. Journal of Business Research, 67(8), 1595-1600. https://doi.org/10.1016/j.jbusres.2013.10.001

Belloti, V., Ambard, A., Turner, D., Gossmann, C., Demkova, K. et Carroll, J. M. (2015, avril). A muddle of models of motivation for using peer-to-peer economy systems. Dans Proceedings of the 33rd Annual ACM Conference on Human Factors in Computing Systems (p. 1085-1094), Séoul, Corée du Sud.

Bouncken, R. B. et Reuschl, A. J. (2018). Coworking-spaces: How a phenomenon of the sharing economy builds a novel trend for the workplace and for entrepreneurship. Review of Managerial Science, 12(1), 317-334. https://doi.org/10.1007/s11846-016-0215-y

Brodie, R. J., Ilic, A., Juric, B. et Hollebeek, L. (2013). Consumer engagement in a virtual brand community: An exploratory analysis. Journal of Business Research, 66(1), 105-114. https://doi.org/10.1016/j.jbusres.2011.07.029

Bucher, E., Fieseler, C. et Lutz, C. (2016). What's mine is yours (for a nominal fee): Exploring the spectrum of utilitarian to altruistic motives for Internet-mediated sharing. Computers in Human Behavior, 62, 316-326. https:/ /doi.org/10.1016/j.chb.2016.04.002

Codagnone, C., Abadie, F. et Biagi, F. (2016). The future of work in the « sharing economy »: Market efficiency and equitable opportunities or unfair precarisation? Séville, Espagne: Institute for Prospective Technological Studies, Joint Research Centre. Repéré à: https://papers.ssrn.com/sol3/papers.cfm?abstract_id=2784774

Cohen, B. et Muñoz, P. (2016). Sharing cities and sustainable consumption and production: Towards an integrated framework. Journal of Cleaner Production, 134, 87-97. https://doi.org/10.1016/j.jclepro.2015.07.133

Dreyer, B., Lüdeke-Freund, F., Hamann, R. et Faccer, K. (2017). Upsides and downsides of the sharing economy: Collaborative consumption business models' stakeholder value impacts and their relationship to context. Technological Forecasting \& Social Change, 125, 87-104. https://doi.org/10.1016/j.techfore.2017.03.036

Edbring, E. G., Lehner, M. et Mont, O. (2016). Exploring consumer attitudes to alternative models of consumption: Motivations and barriers. Journal of Cleaner Production, 123, 5-15. https://doi.org/10.1016/j.jclepro.2015.10.107

Ertz, M., Durif, F. et Arcand, M. (2016). Collaborative consumption: Conceptual snapshot at a buzzword. Journal of Entrepreneurship Education, 19(2), 1-23. http://dx.doi.org/10.2139/ssrn.2799884

Ertz, M., Durif, F. et Arcand, M. (2019). A conceptual perspective on collaborative consumption. AMS Review, 9(1-2), 27-41. https://doi.org/10.1007/s13162-018-0121-3

Ertz, M. et Leblanc-Proulx, S. (2018). Sustainability in the collaborative economy: A bibliometric analysis reveals emerging interest. Journal of Cleaner Production, 196, 1073-1085. https://doi.org/10.1016/j.jclepro.2018.06.095

Ertz, M., Lecompte, A. et Durif, F. (2017). Dual roles of consumers: Towards an insight into collaborative consumption motives. International Journal of Market Research, 59(6), 725-748. https://doi.org/10.2501/IJMR-2017-040

Commission Européenne. (2016a). A European agenda for the collaborative economy (COM/2016/0356 final). Bruxelles, Belgique : Commission Européenne. Repéré à https://secure.ipex.eu/IPEXL-WEB/dossier/document/COM20160356.do

Commission Européenne. (2016b). The use of collaborative platforms: Flash Eurobarometer 438 Report. Bruxelles, Belgique : Commission Européenne. Repéré à https://data.europa.eu/euodp/es/data/dataset/S2112_438_ENG 
Gazzola, P., Vătămănescu, E. M., Andrei, A. G. et Marrapodi, C. (2018). Users' motivation to participate in the sharing economy: Moving from profits toward sustainable development. Corporate Social Responsibility and Environmental Management, 26(4), 741-751. https://doi.org/10.1002/csr.1715

Hamari, J., Sjöklint, M. et Ukkonen, A. (2016). The sharing economy: Why people participate in collaborative consumption. Journal of the Association of Information Science and Technology, 67(9), 2047-2059. https://doi.org/10.1002/asi.23552

Hawlitschek, F., Notheisen, B. et Teubner, T. (2018). The limits of trust-free systems: A literature review on blockchain technology and trust in the sharing economy. Electronic Commerce Research and Applications, 29, 50-63. https://doi.org/10.1016/j.elerap.2018.03.005

Hawlitschek, F., Teubner, T. et Gimpel, H. (2016). Understanding the sharing economy: Drivers and impediments for participation in peer-to-peer rental. Dans Proceedings of the 49th Hawaii International Conference on System Sciences (p. 4782-4789), Kauai, HI.

Hawlitschek, F., Teubner, T. et Gimpel, H. (2018). Consumer motives for peer-to-peer sharing. Journal of Cleaner Production, 204, 144-157. https://doi.org/10.1016/j.jclepro.2018.08.326

Huws, U., Spencer, N. H., Syrdal, D. S. et Holts, K. (2017). Work in the European gig economy: Research results from the UK, Sweden, Germany, Austria, the Netherlands, Switzerland and Italy. Bruxelles, Belgique: Foundation for European Progressive Studies (FEPS). Repéré à https://uhra.herts.ac.uk/bitstream/handle/2299/19922/Huws_U._Spencer_N.H._Syrdal_D.S._Holt_K._2017_.pdf

Kelly, E. et Barrett, A. (2017). Atypical work and Ireland's labour market collapse and recovery. The Economic and Social Review, 48(4), 463-488. Repéré à https://www.esr.ie/article/view/823

$\mathrm{Li}, \mathrm{H}$. et Wen, H. (2019). How is motivation generated in collaborative consumption: Mediation effect in extrinsic and intrinsic motivation. Sustainability, 11(3), 640. https://doi.org/10.3390/su11030640

Lutz, C., Hoffmann, C. P., Bucher, E. et Fieseler, C. (2018). The role of privacy concerns in the sharing economy. Information Communication \& Society, 21(10), 1472-1492. https://doi.org/10.1080/1369118X.2017.1339726

Möhlmann, M. (2015). Collaborative consumption: Determinants of satisfaction and the likelihood of using a sharing economy option again. Journal of Consumer Behaviour, 14(3), 193-207. https://doi.org/10.1002/cb.1512

Nash C., Jarrahi, M. H., Sutherland, W. et Phillips, G. (2018). Digital nomads beyond the buzzword: Defining digital nomadic work and use of digital technologies. Dans G. Chowdhury, J. McLeod, V. Gillet et P. Willett (dir.), Transforming digital worlds (p. 207-217). Cham, Suisse : Springer. https://doi.org/10.1007/978-3-319-78105-1_25

Ozanne, L. K. et Ballantine, P. W. (2010). Sharing as a form of anti-consumption? An examination of toy library users. Journal of Consumer Behaviour, 9(6), 485-498. https://doi.org/10.1002/cb.334

Park, H. et Armstrong, C. M. J. (2019). Is money the biggest driver? Uncovering motives for engaging in online collaborative consumption retail models for apparel. Journal of Retailing and Consumer Services, 51, 42-50. https://doi.org/10.1016/j.jretconser.2019.05.022

Pesole, A., Urzí-Brancati, M. C., Fernández-Macías, E., Biagi, F. et González-Vázquez, I. (2018). Platform workers in Europe: Evidence from the COLLEEM survey. Luxembourg, Luxembourg: Publications Office of the European Union. Repéré à https://www.researchgate.net/profile/Cesira_Urzi_Brancati/publication/326625733_Platform_Workers_in_ Europe_Evidence_from_the_COLLEEM_Survey/links/5b59a7d7458515c4b2495b3a/Platform-Workers-in-Europe-Evidencefrom-the-COLLEEM-Survey.pdf

Spindeldreher, K., Ak, E., Fröhlich, J. et Schlagwein, D. (2019, septembre). Why won't you share? Barriers to participation in the sharing economy. Dans Proceedings of the 25th American Conference on Information Systems (p. 1-10), Lisbonne, Portugal.

Stofberg, N. et Bridoux, F. (2019). Consumers' choice among peer-to-peer sharing platforms: The other side of the coin. Psychology \& Marketing, 36(12), 1176-1195. https://doi.org/10.1002/mar.21265

Sutherland, W. et Jarrahi, M. H. (2018). The sharing economy and digital platforms: A review and research agenda. International Journal of Information Management, 43, 328-341. https://doi.org/10.1016/j.ijinfomgt.2018.07.004

Ter Huurne, M., Ronteltap, A., Corten, R. et Buskens, V. (2017). Antecedents of trust in the sharing economy: A systematic review. Journal of Consumer Behaviour, 16(6), 485-498. https://doi.org/10.1002/cb.1667

Teubner, T. et Flath, C. M. (2019). Privacy in the sharing economy. Journal of the Association of Information Systems, 20(3), 213-242. https://doi.org/ 10.17705/1jais.00534 
Torrent-Sellens, J. (2019). Collaborative behavior and the sharing economy: Pan-European evidence for a new economic approach. Dans B. Orlando (dir.), Strategy and behaviors in the digital economy (p. 1-19). Londres, GB: IntechOpen.

Tussyadiah, I. P. (2015). An exploratory study on drivers and deterrents of collaborative consumption in travel. Dans I. P. Tussyadiah et A. Inversini (dir.), Information and communication technologies in tourism (p. 817-830). Cham, Suisse: Springer.

Tussyadiah, I. P. (2016). Factors of satisfaction and intention to use peer-to-peer accommodation. International Journal of Hospitality Management, 55, 70-80. https://doi.org/10.1016/j.ijhm.2016.03.005

Vătămănescu, E. M. et Pînzaru, F. (2018). Knowledge management in the sharing economy: Cross-sectoral insights into the future of competitive advantage. Cham, Suisse: Springer.

Wachsmuth, D. et Weisler, A. (2018). Airbnb and the rent gap: Gentrification through the sharing economy. Environment and Planning A: Economy and Space, 50(6), 1147-1170. https://doi.org/10.1177/0308518X18778038

Wilhelms, M. P., Henkel, S. et Falk, T. (2017). To earn is not enough: A means-end analysis to uncover peer-provider's participation motives in peer-to-peer car sharing. Technological Forecasting and Social Change, 125, 38-47. https://doi.org/10.1016/ j.techfore. 2017.03 .03 